\title{
Satisfação com a Especialidade entre os Internos da Formação Específica em Portugal
}

\author{
Career Satisfaction of Medical Residents in Portugal
}

Maria João MARTINS ${ }^{1}$, Inês LAÍNS ${ }^{2,3}$, Bruno BROCHADO ${ }^{4}$, Manuel OLIVEIRA-SANTOS ${ }^{5}$, Pedro PINTO TEIXEIRA ${ }^{6}$, Mariana BRANDÃO ${ }^{7}$, Rui João CERQUEIRA ${ }^{8,9}$, Ricardo CASTRO-FERREIRA ${ }^{10}$, Carlos BERNARDES ${ }^{11}$, Miguel NOBRE MENEZES ${ }^{12}$, Bernardo SOARES BAPTISTA ${ }^{13}$, Ricardo LADEIRAS-LOPES ${ }^{14,15}$, Mariana CRUZ REI ${ }^{16}$, Gilberto PIRES DA ROSA $^{17}$, José Luis MARTINS ${ }^{18}$, Maria MENDONÇA SANCHES ${ }^{19}$, Manuel J. FERREIRA-PINTO ${ }^{8,20}$, Margarida RATO ${ }^{21}$, Miguel COSTAE SILVA ${ }^{22}$, Catarina POLICIANO ${ }^{23}$, João BEATO ${ }^{24}$, João BARBOSA-BREDA ${ }^{24}$, João PIMENTEL TORRES ${ }^{25,26}$, Inês LEAL ${ }^{27,28}$, Sílvia AGUIAR ROSA ${ }^{11}$, Bárbara CARVALHO RIBEIRO ${ }^{29}$, Francisco REGO COSTA ${ }^{30}$, Carolina PALMELA $^{31}$, Tiago CÚRDIA GONÇALVES ${ }^{32}$, Luis MORAIS ${ }^{4}$, Tiago REIS MARQUES ${ }^{33}$

Acta Med Port 2015 Mar-Apr;28(2):209-221

\section{RESUMO}

Introdução: A satisfação com a profissão médica tem sido apontada como um fator essencial para a qualidade assistencial, o bemestar dos doentes e a estabilidade dos sistemas de saúde. Estudos recentes têm vindo a enfatizar um crescente descontentamento dos médicos, principalmente como consequência das alterações das relações laborais.

Objetivos: Avaliar a perceção dos médicos de formação específica em Portugal, sobre as expectativas e grau de satisfação com a profissão, especialidade e local de formação; razões da insatisfação e intenção de emigrar.

Material e Métodos: Estudo transversal. A colheita de dados foi efetuada entre Maio e Agosto de 2014 através de um Inquérito online sobre a "Satisfação com a Especialidade".

Resultados: De uma população total de 5788 médicos, foram obtidas 804 respostas (12,25\% do total de médicos internos). Desta amostra, $77 \%$ das respostas correspondem a internos dos três primeiros anos de formação. Verificou-se que $90 \%$ dos médicos se encontram satisfeitos com a especialidade, tendo-se encontrado também níveis elevados de satisfação com a profissão (85\%) e local de formação (86\%). Por outro lado, constatou-se que estes diminuíam com a progressão ao longo dos anos de internato. A avaliação global sobre o panorama da prática médica foi negativa e $65 \%$ dos médicos responderam que consideram emigrar após conclusão do internato.

Conclusão: Os médicos internos em Portugal apresentam níveis positivos de satisfação com a sua profissão. No entanto, a sua opinião sobre o panorama da Medicina e os resultados relativos à intenção de emigrar alertam para a necessidade de tomada de medidas para inverter este cenário.

Palavras-chave: Internato; Medicina; Portugal; Questionários; Satisfação Profissional.

1. Faculdade de Psicologia e Ciências da Educação. Universidade de Coimbra. Coimbra. Portugal.

2. Serviço de Oftalmologia. Centro Hospitalar e Universitário de Coimbra. Coimbra. Portugal.

3. Faculdade de Medicina da Universidade de Coimbra. Coimbra. Portugal.

4. Serviço de Cardiologia. Centro Hospitalar do Porto. Porto. Portugal.

5. Serviço de Cardiologia A. Centro Hospitalar e Universitário de Coimbra. Coimbra. Portugal.

6. Serviço de Cardiologia. Centro Hospitalar de Lisboa Central. Lisboa. Portugal.

7. Serviço de Oncologia Médica. Instituto Português de Oncologia Francisco Gentil. Porto. Portugal.

8. Serviço de Cirurgia Cardiotorácica. Centro Hospitalar São João. Porto. Portugal.

9. Departamento de Fisiologia e Cirurgia Cardiotorácica. Faculdade de Medicina da Universidade do Porto. Porto. Portugal.

10. Serviço de Cirurgia Vascular. Centro Hospitalar de São João. Porto. Portugal.

11. Serviço de Gastroenterologia. Hospital de Santo António dos Capuchos. Centro Hospitalar de Lisboa Central. Lisboa. Portugal.

12. Serviço de Cardiologia. Hospital de Santa Maria. Centro Hospitalar de Lisboa Norte. Lisboa. Portugal.

13. Serviço de Medicina. Hospital da Luz. Lisboa. Portugal.

14. Serviço de Cardiologia. Centro Hospitalar de Vila Nova de Gaia/Espinho. Vila Nova de Gaia. Portugal.

15. Unidade de Investigação Cardiovascular. Faculdade de Medicina. Universidade do Porto. Porto. Portugal.

16. Serviço de Neurologia. Centro Hospitalar Entre o Douro e Vouga. Santa Maria da Feira. Portugal.

17. Serviço de Medicina Interna. Centro Hospitalar de São João. Porto. Portugal.

18. Serviço de Cardiologia. Centro Hospitalar do Baixo Vouga. Aveiro. Portugal.

19. Serviço de Dermatologia. Hospital de Santa Maria. Centro Hospitalar de Lisboa Norte. Lisboa. Portugal.

20. Serviço de Oncologia Cirúrgica. Instituto Português de Oncologia Francisco Gentil. Porto. Portugal.

21. Serviço de Dermatovenerologia. Hospital Distrital de Santarém. Santarém. Portugal.

22. Serviço de Dermatovenerologia. Centro Hospitalar de São João. Porto. Portugal.

23. Departamento de Ginecologia e Obstetrícia. Hospital de Santa Maria. Centro Hospitalar Lisboa Norte. Lisboa. Portugal.

24. Departamento de Oftalmologia. Centro Hospitalar de São João. Porto. Portugal.

25. Serviço de Urologia. Hospital de Braga. Braga. Portugal.

26. Instituto de Ciências da Vida e da Saúde. Universidade do Minho. Braga. Portugal.

27. Departamento de Ética e Deontologia Médica. Faculdade de Medicina de Lisboa. Lisboa. Portugal.

28. Serviço de Oftalmologia. Hospital de Santa Maria. Centro Hospitalar Lisboa Norte. Lisboa. Portugal.

29. Serviço de Ginecologia/Obstetrícia. Hospital de Braga. Braga. Portugal.

30. Serviço de Radiologia. Hospital de São João. Porto. Portugal.

31. Serviço de Gastroenterologia. Hospital Beatriz Ângelo. Loures. Portugal.

32. Serviço de Gastroenterologia. Centro Hospitalar do Alto Ave. Guimarães. Portugal.

33. Department of Psychosis Studies. Institute of Psychiatry, Psychology and Neuroscience. King's College. London. United Kingdom.

Recebido: 13 de Outubro de 2014 - Aceite: 16 de Fevereiro de 2015 | Copyright $\odot$ Ordem dos Médicos 2015 


\section{ABSTRACT}

Introduction: The satisfaction with the medical profession has been identified as an essential factor for the quality of care, the wellbeing of patients and the healthcare systems' stability. Recent studies have emphasized a growing discontent of physicians, mainly as a result of changes in labor relations.

Objectives: To assess the perception of Portuguese medical residents about: correspondence of residency with previous expectations; degree of satisfaction with the specialty, profession and place of training; reasons for dissatisfaction; opinion regarding clinical practice in Portugal and emigration intents.

Material and Methods: Cross-sectional study. Data collection was conducted through the "Satisfaction with Specialization Survey", created in an online platform, designed for this purpose, between May and August 2014.

Results: From a total population of 5788 medical residents, $804(12.25 \%)$ responses were obtained. From this sample, $77 \%$ of the responses were from residents in the first three years. Results showed that $90 \%$ of the residents are satisfied with their specialty, $85 \%$ with the medical profession and $86 \%$ with their place of training. Nevertheless, results showed a decrease in satisfaction over the final years of residency. The overall assessment of the clinical practice scenario in Portugal was negative and $65 \%$ of residents have plans to emigrate after completing their residency.

Conclusion: Portuguese residents revealed high satisfaction levels regarding their profession. However, their views on Portuguese clinical practice and the results concerning the intent to emigrate highlight the need to take steps to reverse this scenario.

Keywords: Internship and Residency; Job Satisfaction; Medicine; Portugal; Questionnaires.

\section{INTRODUÇÃO}

A profissão médica é ainda uma das carreiras mais atrativas em Portugal. De facto, ano após ano, observa-se que o curso de Medicina é aquele com maior procura e de mais difícil acesso no Ensino Superior. Para isto contribui não só a perceção da opinião pública sobre a Medicina, mas também a perceção sobre as vantagens sociais e financeiras associadas a esta profissão. Torna-se portanto expectável que os médicos sejam profissionais realizados e satisfeitos com a sua vida profissional.

Hoje é reconhecido que a satisfação dos médicos com a sua profissão tem implicações não só ao nível do indivíduo, mas também na saúde dos seus doentes e em termos de Saúde Pública. Os doentes de médicos que se consideram muito ou extremamente satisfeitos com o seu trabalho têm maiores níveis de contentamento com os cuidados médicos que receberam do que aqueles tratados por médicos insatisfeitos. ${ }^{1}$ Por outro lado, médicos insatisfeitos têm mais problemas de saúde, maior probabilidade de ausências laborais injustificadas ${ }^{2}$ e de sofrerem de mais problemas psicológicos e mesmo burnout. ${ }^{3}$ Em termos de saúde pública, a necessidade de manter um correto equilíbrio entre todas as especialidades médicas é fundamental para manter um sistema de saúde com elevados padrões de qualidade. Este balanço tem vindo a ser alterado com o êxodo de médicos de especialidades menos atrativas para outras percebidas como mais vantajosas. ${ }^{4,5}$ Também no panorama português assiste-se anualmente a especialidades em que as vagas não são ocupadas na totalidade ou a mudanças de especialidade, comprometendo o futuro dessas especialidades e os cuidados de saúde em Portugal.

Vários estudos internacionais mostram, no entanto, que a satisfação profissional na comunidade médica é elevada, com aproximadamente $85 \%$ dos médicos moderadamente ou muito satisfeitos com a sua profissão. ${ }^{6,7}$ Esta satisfação é observada quer entre internos ${ }^{8,9}$ quer entre especialistas. ${ }^{10,11}$ No entanto, as diferenças entre especialidades são significativas, sendo que no maior estudo feito até à data são as especialidades de Doenças Infecciosas, Dermatologia e Pediatria as que geram maior satisfação entre os seus profissionais, enquanto outras especialidades como Ginecologia, Pneumologia e Otorrinolaringologia se encontram no extremo oposto. ${ }^{12}$

No entanto, nos últimos anos tem-se vindo a assistir a uma redução da satisfação com a profissão médica, consequência de alterações no prestígio da profissão, perda de autonomia, aumento do trabalho administrativo e do horário de trabalho e redução da remuneração salarial. De facto, num estudo de 2001, 58\% entre 2608 médicos americanos referiram que o seu entusiasmo com a Medicina diminuiu nos cinco anos prévios, enquanto que $87 \%$ apontou para uma redução da sua perceção de bem-estar nesse período. ${ }^{13}$ É também de esperar que fatores conjecturais, como a remuneração salarial e as condições de trabalho afetem a satisfação profissional. A importância do ambiente económico foi comprovada num estudo recente, onde a satisfação dos médicos noruegueses foi comparada com a dos seus colegas islandeses, a viverem uma recessão profunda após a crise económica de 2008. ${ }^{14}$ Nesse estudo a satisfação dos médicos islandeses era significativamente menor que a dos pares noruegueses, já controlando para fatores individuais e relacionados com o trabalho. Os mesmos autores, num outro artigo, procuraram perceber o impacto que a mesma crise teve sobre a migração dos médicos islandeses. Duma amostra de 465 médicos, correspondendo a $55 \%$ de todos os médicos especialistas islandeses, $63 \%$ dos médicos consideravam emigrar num breve período temporal. ${ }^{15}$

Apesar da sua importância e relevância, a satisfação profissional nunca foi avaliada entre os internos de formação específica em Portugal. Neste estudo procurou-se pela primeira vez avaliar esta satisfação, bem como analisar as diferenças entre especialidades.

\section{MATERIAL E MÉTODOS Participantes}

Os médicos convidados para participar neste estudo foram os médicos em formação específica que constam da base de dados do projeto Exame da Especialidade (www. examedaespecialidade.com), que forneceu os referidos contactos. Esta base de dados contém 5788 médicos internos, correspondendo a $88,19 \%$ do total de 6563 médicos a frequentar o internato médico em Portugal. $O$ inquérito foi 
enviado por correio eletrónico e um total de 804 médicos internos concordaram participar tendo respondido ao inquérito. Este número corresponde a uma taxa de resposta de $12,25 \%$ do total de internos em formação em Portugal.

\section{Instrumentos}

\section{Inquérito de satisfação com a especialidade}

Este instrumento foi construído especificamente para o presente estudo, sendo o seu modelo e questões semelhantes às publicadas em outros estudos que avaliaram a satisfação com a especialidade.$^{7,8,10-13} \mathrm{O}$ inquérito consistiu em 15 questões dicotómicas (Sim/Não) ou utilizando uma escala de Lickert de 10 pontos. Esta escala numérica permitiu classificar os resultados em cinco grupos: Não Corresponde Nada/Nada Satisfeito = 1 e 2; Corresponde Pouco/Pouco Satisfeito = 3 e 4; Neutro $=5$ e 6; Corresponde Muito/Muito Satisfeito $=7$ e 8; Corresponde Totalmente/Extremamente Satisfeito $=9$ e 10. Este questionário avaliou a perceção subjetiva do médico em relação à sua especialidade em termos de: correspondência das expectativas à realidade; grau de satisfação; razões da insatisfação; satisfação com o local de formação; satisfação com a profissão; prática da Medicina; intenção de emigração. O envio do inquérito foi realizado através de correio electrónico, utilizando o software SurveyMonkey ${ }^{\circledR}$, que permite uma única resposta por utilizador eliminando desta forma qualquer duplicação de resultados.

\section{Procedimento e análise estatística}

A população em análise consistiu nos médicos que se encontram a realizar o internato em Portugal. Para controlar possíveis vieses, foram realizadas análises por ano de internato, por especialidade e por local de formação. De forma a garantir o rigor estatístico e a representatividade dos resultados, foram realizadas análises apenas para as especialidades que contaram com 10 ou mais respostas, tendo sido utilizado o mesmo critério para as análises por local de formação. Em relação a esta última, de forma a que se pudesse eliminar a influência que a insatisfação com a especialidade pudesse ter sobre a avaliação da satisfação com o local de formação, realizou-se uma análise ajustada a este factor apenas com os médicos que pontuaram igual ou superior a 5 na satisfação com a especialidade (valor que indica uma satisfação mínima com a especialidade, máximo $=10$ ). Foi ainda analisada a subpopulação de médicos cuja especialidade foi a primeira escolha. A análise estatística foi realizada com recurso ao software SPSS versão 20.0 .

\section{RESULTADOS}

\section{Amostra}

O número de médicos por especialidade e por local de formação podem ser analisados na Tabela 1a e $1 \mathrm{~b}$. Relativamente à distribuição por ano de internato, verificase que a maioria das respostas obtidas correspondiam a médicos nos primeiros anos da formação, com $32 \%$ ( $n=$ 238) encontrando-se a frequentar o primeiro ano de inter- nato, $27 \%(n=217)$ no segundo, $18 \%(n=143)$ no terceiro, $15 \%(n=118)$ no quarto, $5 \%(n=37)$ no quinto e $0,2 \%$ $(n=2)$ no sexto ano.

\section{Resultados globais \\ Escolha da especialidade}

Para a grande maioria a especialidade em que se encontram foi a sua primeira escolha $(n=611 ; 81 \%)$, enquanto que $19 \%$ ( $n=191)$ entraram numa especialidade que foi a sua segunda opção. Quando questionados se, caso tivessem oportunidade, escolheriam de novo a mesma especialidade, $85 \%$ referiu que $\operatorname{sim}(n=632 ; 85 \%)$. Numa sub-análise incluindo apenas os médicos em que a especialidade em que se encontram não foi a sua primeira opção ( $n=191)$, verifica-se que $57 \%(n=109)$ destes não mudaria atualmente de especialidade.

\section{Expectativas em relação à especialidade.}

A grande maioria dos internos considera que a especialidade em que se encontram corresponde muito ou mesmo totalmente ao esperado (Fig. 1A). Os resultados por ano de internato mostram que a realidade da especialidade corresponde às expectativas em todos os anos do internato analisados. No entanto, são os médicos do $1^{\circ}$ ano do internato os que consideram as suas expectativas mais próximas da realidade $(M=4,28 ; D P=0,83)$, decrescendo os valores ao longo do internato (Fig. 1A).

\section{Grau de satisfação com a especialidade}

A maioria dos médicos indicou estar extremamente ou muito satisfeito com a sua especialidade e somente $10 \%$ dos médicos referem estar pouco satisfeitos ou muito insatisfeitos (Fig. 1B). Quando analisados por ano de internato, verifica-se uma diminuição da satisfação ao longo do internato $\left(1^{\circ}\right.$ ano $-M=4,29, D P=1 ; 2^{\circ}$ ano $-M=4,11$, DP = 1,$13 ; 3^{\circ}$ ano $-M=4,06$, $D P=1,01 ; 4^{\circ}$ ano $-M=4,02$, DP $=1,09 ; 5^{\circ}$ ano $-M=3,73$, DP = 1,36). Quando questionados acerca das razões da insatisfação, $47 \%$ apontaram as condições de exercício da prática clínica como a principal razão de insatisfação, $23 \%$ indicaram a realidade da especialidade, $16 \%$ o local de formação e em $14 \%$ dos casos outras razões.

\section{Grau de satisfação com o local de formação}

A maioria dos médicos encontra-se muito satisfeito ou extremamente satisfeito com o local escolhido para a realização da formação específica (Fig. 1C), enquanto que apenas $14 \%$ afirmou encontrar-se pouco satisfeito ou muito insatisfeito com o local de formação. Com a progressão do internato médico verificou-se uma diminuição da satisfação com o local de especialidade.

\section{Grau de satisfação com a profissão}

Quando questionados se voltariam a escolher o curso de Medicina, a grande maioria das respostas foi positiva ( $\mathrm{N}$ $=632 ; 85 \%$ ), mas verifica-se uma diminuição dessa intenção ao longo do internato ( $1^{\circ}$ ano: $92,2 \% ; 2^{\circ}$ ano: $75,4 \% ; 3^{\circ}$ 
Tabelas 1A - Número de médicos colocados por especialidade, número de respostas e respectivas taxas de resposta

\begin{tabular}{|c|c|c|c|}
\hline Especialidade & $\begin{array}{c}\text { Número de Médicos } \\
\text { Colocados }\end{array}$ & $\begin{array}{l}\text { Número de } \\
\text { Respostas }\end{array}$ & Taxa de Resposta \\
\hline Medicina Do Trabalho & 10 & 4 & $40,00 \%$ \\
\hline Medicina Desportiva & 5 & 2 & $40,00 \%$ \\
\hline Medicina Legal & 32 & 11 & $34,38 \%$ \\
\hline Cirurgia Cardíaca & 13 & 4 & $30,77 \%$ \\
\hline Dermatovenereologia & 36 & 8 & $22,22 \%$ \\
\hline Gastrenterologia & 106 & 22 & $20,75 \%$ \\
\hline Radiodiagnóstico & 114 & 22 & $19,30 \%$ \\
\hline Psiquiatria da Infância e da Adolescência & 54 & 9 & $16,67 \%$ \\
\hline Infecciologia & 70 & 11 & $15,71 \%$ \\
\hline Ginecologia / Obstetrícia & 209 & 32 & $15,31 \%$ \\
\hline Cardiologia & 128 & 18 & $14,06 \%$ \\
\hline Estomatologia & 36 & 5 & $13,89 \%$ \\
\hline Urologia & 51 & 7 & $13,73 \%$ \\
\hline Pediatria Médica & 333 & 45 & $13,51 \%$ \\
\hline Medicina Geral e Familiar & 1674 & 220 & $13,14 \%$ \\
\hline Ortopedia & 199 & 25 & $12,56 \%$ \\
\hline Anatomia Patológica & 80 & 10 & $12,50 \%$ \\
\hline Cirurgia Geral & 345 & 42 & $12,17 \%$ \\
\hline Neurologia & 107 & 13 & $12,15 \%$ \\
\hline Pneumologia & 107 & 13 & $12,15 \%$ \\
\hline Medicina Física e Reabilitação & 124 & 15 & $12,10 \%$ \\
\hline Nefrologia & 85 & 10 & $11,76 \%$ \\
\hline Cirurgia Plástica Reconstrutiva & 43 & 5 & $11,63 \%$ \\
\hline Oncologia Medica & 161 & 18 & $11,18 \%$ \\
\hline Psiquiatria & 251 & 28 & $11,16 \%$ \\
\hline Anestesiologia & 329 & 35 & $10,64 \%$ \\
\hline Angiologia e Cirurgia Vascular & 49 & 5 & $10,20 \%$ \\
\hline Saúde Pública & 148 & 15 & $10,14 \%$ \\
\hline Otorrinolaringologia & 100 & 10 & $10,00 \%$ \\
\hline Radioterapia & 40 & 4 & $10,00 \%$ \\
\hline Endocrinologia e Nutrição & 63 & 6 & $9,52 \%$ \\
\hline Oftalmologia & 117 & 11 & $9,40 \%$ \\
\hline Imunohemoterapia & 75 & 7 & $9,33 \%$ \\
\hline Medicina Interna & 957 & 87 & $9,09 \%$ \\
\hline Hematologia Clinica & 83 & 7 & $8,43 \%$ \\
\hline Reumatologia & 48 & 4 & $8,33 \%$ \\
\hline Cirurgia Pediátrica & 24 & 2 & $8,33 \%$ \\
\hline Neuroradiologia & 45 & 3 & $6,67 \%$ \\
\hline Cardiologia Pediátrica & 18 & 1 & $5,56 \%$ \\
\hline Genética Médica & 18 & 1 & $5,56 \%$ \\
\hline Medicina Nuclear & 19 & 1 & $5,26 \%$ \\
\hline Neurocirurgia & 46 & 2 & $4,35 \%$ \\
\hline Cirurgia Torácica & 28 & 1 & $3,57 \%$ \\
\hline Imunoalergologia & 42 & 1 & $2,38 \%$ \\
\hline Patologia Clínica & 104 & 2 & $1,92 \%$ \\
\hline Cirurgia Maxilo-facial & 21 & 0 & $0,00 \%$ \\
\hline
\end{tabular}


Tabelas 1B - Número de respostas por Hospital e respectivas taxas de respostae respetivas taxas de resposta

\begin{tabular}{|c|c|c|}
\hline Hospital & Respostas & $\begin{array}{l}\text { Percentagem sobre as } \\
\text { respostas totais }\end{array}$ \\
\hline Centro Hospitalar de São João & 46 & $9,40 \%$ \\
\hline Centro Hospitalar e Universitário de Coimbra & 44 & $9,00 \%$ \\
\hline Centro Hospitalar de Lisboa Central & 43 & $8,80 \%$ \\
\hline Centro Hospitalar de Lisboa Norte & 41 & $8,40 \%$ \\
\hline Centro Hospitalar do Porto & 37 & $7,50 \%$ \\
\hline Centro Hospitalar de Lisboa Ocidental & 26 & $5,30 \%$ \\
\hline Centro Hospitalar de Vila Nova de Gaia-Espinho & 21 & $4,30 \%$ \\
\hline Hospital Garcia de Orta & 18 & $3,70 \%$ \\
\hline Hospital Prof. Doutor Fernando Fonseca & 17 & $3,50 \%$ \\
\hline Hospital de Braga & 16 & $3,30 \%$ \\
\hline Centro Hospitalar Algarve & 14 & $2,90 \%$ \\
\hline ULS Matosinhos & 14 & $2,90 \%$ \\
\hline IPO Lisboa & 11 & $2,20 \%$ \\
\hline Centro Hospitalar Alto Ave & 10 & $2,00 \%$ \\
\hline Centro Hospitalar do Baixo Vouga & 9 & $1,80 \%$ \\
\hline IPO Porto & 8 & $1,60 \%$ \\
\hline Centro Hospitalar de Leiria & 7 & $1,40 \%$ \\
\hline Centro Hospitalar de Tondela-Viseu & 7 & $1,40 \%$ \\
\hline Centro Hospitalar de Setúbal & 6 & $1,20 \%$ \\
\hline Centro Hospitalar Trás-os-Montes e Alto Douro & 6 & $1,20 \%$ \\
\hline Centro Hospitalar Psiquiátrico de Lisboa & 6 & $1,20 \%$ \\
\hline Hospital Espírito Santo Évora & 6 & $1,20 \%$ \\
\hline Hospital Dr. Nélio Mendonça - Funchal & 6 & $1,20 \%$ \\
\hline ULS Nordeste & 6 & $1,20 \%$ \\
\hline ULS Alto Minho & 6 & $1,20 \%$ \\
\hline Centro Hospitalar Barreiro Montijo & 5 & $1,00 \%$ \\
\hline Centro Hospitalar Cova da Beira & 5 & $1,00 \%$ \\
\hline Centro Hospitalar de Entre o Douro e Vouga & 5 & $1,00 \%$ \\
\hline Hospital Distrital de Santarém & 5 & $1,00 \%$ \\
\hline ULS Baixo Alentejo & 5 & $1,00 \%$ \\
\hline Hospital Beatriz Ângelo & 4 & $0,80 \%$ \\
\hline Hospital Divino Espírito Santo Ponta Delgada & 4 & $0,80 \%$ \\
\hline Hospital Vila Franca de Xira & 4 & $0,80 \%$ \\
\hline HPP Cascais & 3 & $0,60 \%$ \\
\hline Centro Hospitalar do Tâmega e Sousa & 3 & $0,60 \%$ \\
\hline IPO Coimbra & 3 & $0,60 \%$ \\
\hline Cuf Descobertas & 2 & $0,40 \%$ \\
\hline Hospital da Horta & 2 & $0,40 \%$ \\
\hline Hospital de Magalhães Lemos & 2 & $0,40 \%$ \\
\hline ULS Norte Alentejano & 2 & $0,40 \%$ \\
\hline ULS Guarda & 2 & $0,40 \%$ \\
\hline Hospital de Santo Espírito de Angra do Heroísmo & 1 & $0,20 \%$ \\
\hline Hospital Santa Maria Maior - Barcelos & 1 & $0,20 \%$ \\
\hline ULS Castelo Branco & 1 & $0,20 \%$ \\
\hline ULS Litoral Alentejano & 1 & $0,20 \%$ \\
\hline
\end{tabular}




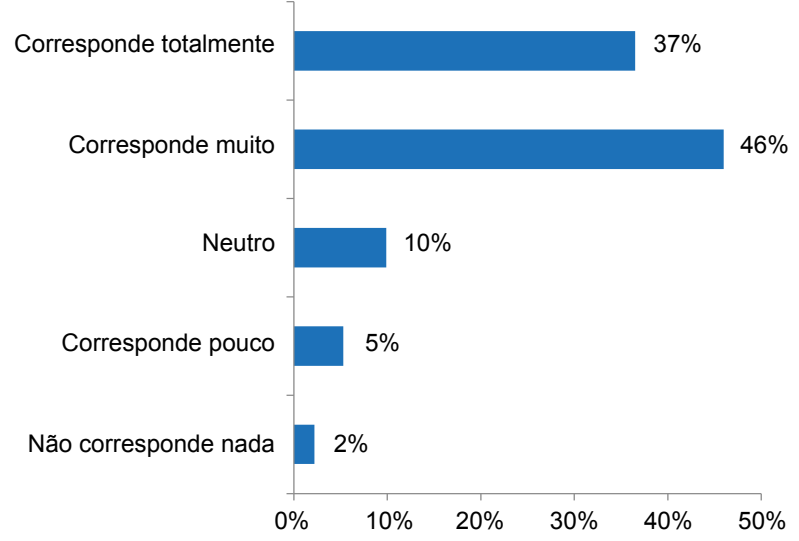

Figura $1 \mathrm{~A}$ - Expectativas em relação à especialidade

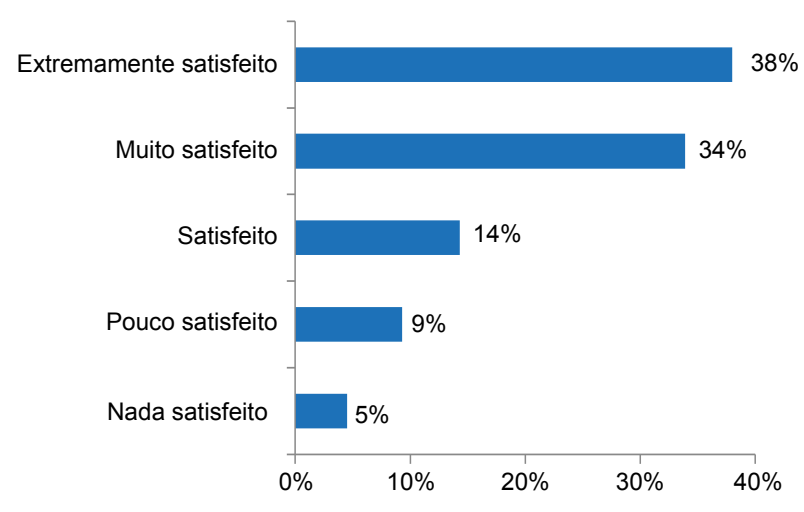

Figura 1C - Grau de satisfação com o local de formação

ano: $72,5 \% ; 4^{\circ}$ ano: $75,9 \% ; 5^{\circ}$ ano: $\left.80 \%\right)$.

\section{Panorama da prática médica em Portugal}

A maioria dos médicos acha que existiu uma deterioração da prática médica, nenhum médico considerando que houve uma melhoria significativa (Fig. 1D). Quando analisados por ano de internato, há uma progressão negativa com o avançar do internato.

\section{Emigração e procura de trabalho fora de Portugal}

Sessenta e cinco por cento $(n=484)$ dos médicos consideram emigrar e procurar trabalho fora de Portugal quando terminarem a especialidade. Quando analisado por ano de internato, observa-se um aumento da taxa de médicos que pretende emigrar ao longo do internato. Assim, enquanto que no $1^{\circ}$ ano somente $53 \%$ considera emigrar $\left(2^{\circ}\right.$ ano $-62 \% ; 3^{\circ}$ ano $-76 \%$ e $4^{\circ}$ ano - $73 \%$ ) já no último ano de internato cerca de $75 \%$ dos médicos internos considera esta hipótese. As razões mais apontadas como justificação foram as razões financeiras ( $41 \%$ ) a falta de oportunidades de trabalho $(31 \%)$. Cerca de $20 \%$ dos médicos selecionou

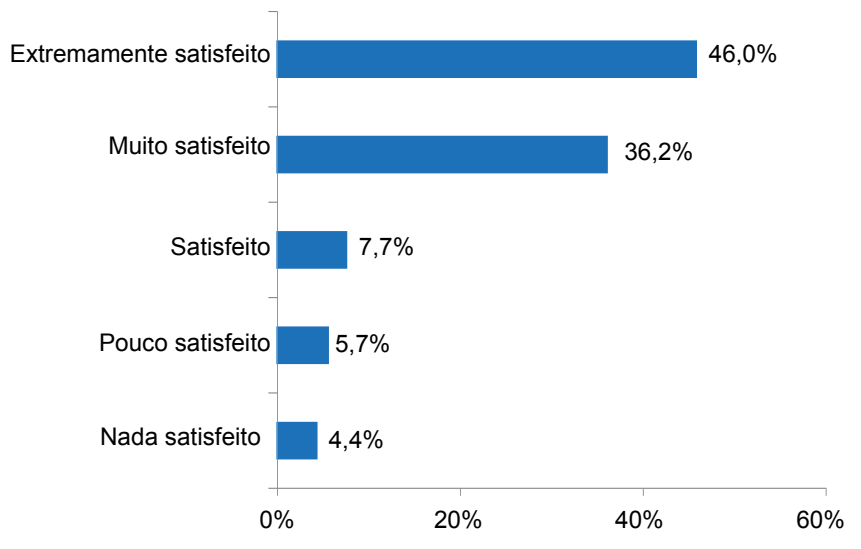

Figura 1B - Grau de satisfação com a especialidade

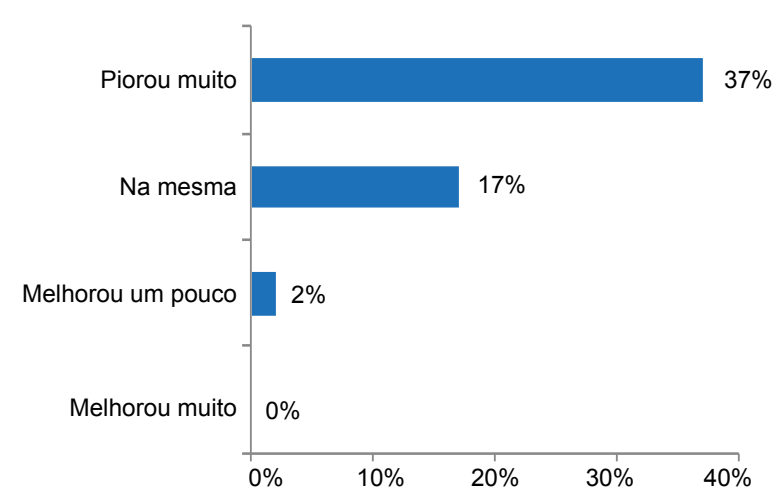

Figura 1D - Panorama da prática médica em Portugal

a opção "outro", que na maioria dos casos se refere à conjugação de várias destas hipóteses, e 10\% refere a falta de emprego.

\section{Resultados por especialidade}

Com o objetivo de perceber a variação nas questões dependendo da especialidade em que os médicos se encontram, realizaram-se sub-análises por especialidade. Num universo de 47 especialidades médicas em Portugal, foram analisadas somente aquelas em que se obtiveram pelo menos 10 respostas. Assim, analisaram-se somente 22 das 47 especialidades existentes.

\section{Expectativas em relação à especialidade}

Apesar de diferenças importantes entre as especialidades, todas pontuam em média acima de 5 , mostrando que os resultados são globalmente positivos, sendo a Anestesiologia, Ginecologia/Obstetrícia e Otorrinolaringologia aquelas onde médicos acham que a realidade mais corresponde ao que esperavam (Fig. 2A). 


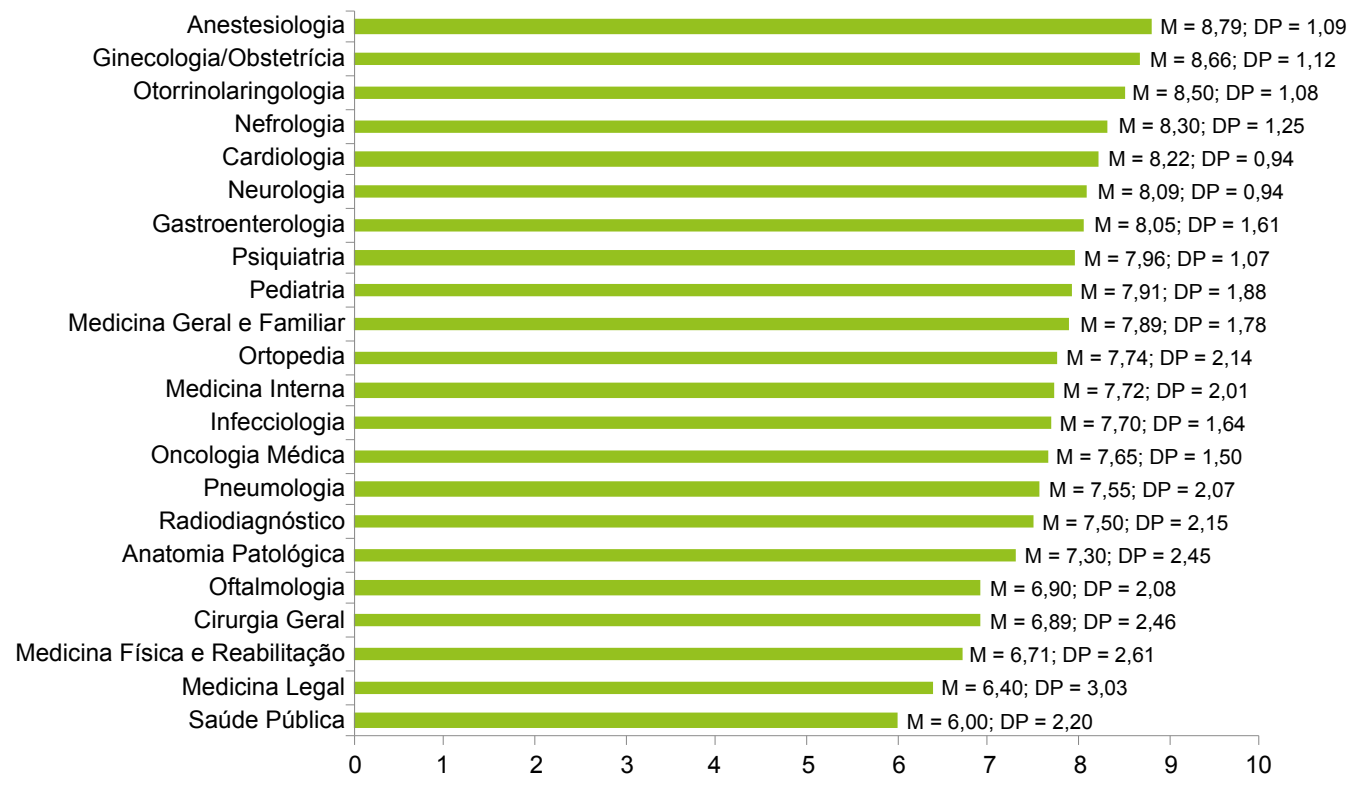

Figura 2A - Expectativas em relação à especialidade

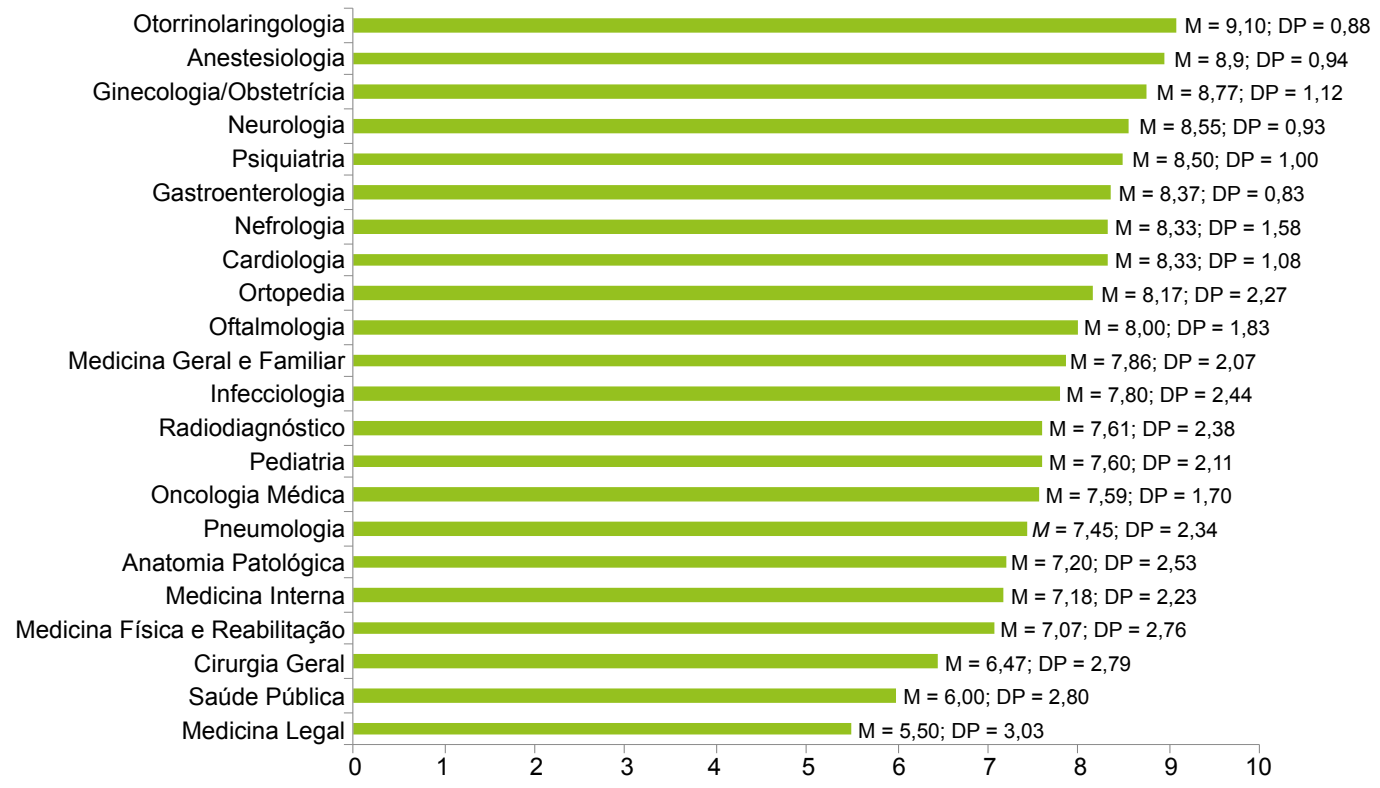

Figura 2B - Grau de satisfação com a especialidade

\section{Grau de satisfação com a especialidade}

São os médicos de Otorrinolaringologia ( $M=9,1 ; \mathrm{DP}=$ $0,88)$, Anestesiologia ( $M=8,97$; $D P=0,94)$ e Ginecologia/ Obstetrícia ( $M=8,77 ; D P=1,12)$ que reportam um grau de satisfação mais elevado. Níveis mais baixos encontram-se na Medicina Legal $(M=5,5 ; D P=3,03)$, Saúde Pública ( $M$ $=6 ; \mathrm{DP}=2,80)$ e a Cirurgia Geral $(M=6,47 ; \mathrm{DP}=2,79)$.

\section{Escolha da especialidade}

Quando questionados se escolheriam novamente a mesma especialidade, foram os médicos de Otorrinola- ringologia, Psiquiatria e Anestesiologia aqueles que mais referiram que permaneceriam na mesma especialidade. Já na Saúde Pública, Cirurgia Geral e Medicina Legal observou-se um maior número de médicos a ponderar escolher outra especialidade se lhes fosse dada essa oportunidade (Tabela 2A).

Perspetivas de futuro e procura de trabalho fora de Portugal

São os Médicos de Anestesiologia aqueles que consideram que a sua especialidade Ihes dá mais perspetivas 
Tabelas 2A - Escolha de novo da mesma especialidade

\begin{tabular}{|c|c|c|}
\hline Especialidade & Sim & Não \\
\hline Otorrinolaringologia & $100 \%$ & - \\
\hline Psiquiatria & $100 \%$ & - \\
\hline Anestesiologia & $97 \%$ & $3 \%$ \\
\hline Cardiologia & $94 \%$ & $6 \%$ \\
\hline Radiodiagnóstico & $94 \%$ & $6 \%$ \\
\hline Ginecologia/Obstetrícia & $91 \%$ & $9 \%$ \\
\hline Neurologia & $90 \%$ & $10 \%$ \\
\hline Medicina Geral e Familiar & $89 \%$ & $11 \%$ \\
\hline Oncologia Médica & $88 \%$ & $12 \%$ \\
\hline Medicina Interna & $85 \%$ & $15 \%$ \\
\hline Pediatria & $84 \%$ & $16 \%$ \\
\hline Gastrenterologia & $84 \%$ & $16 \%$ \\
\hline Ortopedia & $83 \%$ & $17 \%$ \\
\hline Anatomia Patológica & $80 \%$ & $20 \%$ \\
\hline Infecciologia & $80 \%$ & $20 \%$ \\
\hline Oftalmologia & $80 \%$ & $20 \%$ \\
\hline Medicina Física e Reabilitação & $79 \%$ & $21 \%$ \\
\hline Pneumologia & $73 \%$ & $27 \%$ \\
\hline Nefrologia & $70 \%$ & $30 \%$ \\
\hline Saúde Pública & $67 \%$ & $33 \%$ \\
\hline Cirurgia Geral & $61 \%$ & $39 \%$ \\
\hline Medicina Legal & $60 \%$ & $40 \%$ \\
\hline Total & $85 \%$ & $15 \%$ \\
\hline
\end{tabular}

de futuro, seguida da Oncologia Médica e Medicina Geral e Familiar. Por sua vez, somente a Pediatria pontua abaixo do cut-off de 5 valores (Fig. 3). Quando questionados acerca da intenção de emigrar são os médicos de Medicina Geral e Familiar, Medicina Legal e Saúde Pública aqueles que menos consideram emigrar, com os internos de Cardiologia, Anatomia Patológica e Infecciologia com maior propensão de emigrar (Tabela 2B).

\section{Resultados por local de formação}

Com o objetivo de perceber a variação na satisfação com o local de formação, realizaram-se sub-análises por Hospital. Por razões estatísticas, não foram analisadas as USF uma vez que têm um número de médico internos relativamente baixo o que impede uma análise estatística robusta.

\section{Grau de satisfação com o local de formação}

São os grandes Centros Hospitalares $(\mathrm{CH})$, como o CH Universitário de Coimbra, o CH São João e o CH Lisboa Norte, aqueles onde os médicos apresentam menor satis- fação, enquanto que com maiores índices de satisfação surgem o CH Vila Nova de Gaia e o Hospital Prof. Dr. Fernando Fonseca (Fig. 4). De forma a controlar o efeito que a satisfação com a especialidade poderá ter na satisfação com o local de formação, foi realizada uma sub-análise ajustada a essa variável, considerando apenas os médicos cuja satisfação foi igual ou superior a 5 (numa escala de 1 a 10). São os Hospitais de Braga, CH Vila Nova de Gaia/ Espinho e o Hospital Prof. Dr. Fernando Fonseca em que houve maior satisfação, enquanto que no extremo oposto se encontra o $\mathrm{CH}$ São João, $\mathrm{CH}$ do Algarve e o CH Universitário de Coimbra (Fig. 5).

Resultados para a subamostra de médicos cuja atual especialidade foi a primeira escolha

Com o objetivo de controlar o facto de a especialidade não ter sido a primeira escolha, realizaram-se as análises tendo em conta apenas os médicos cuja especialidade atual foi a primeira escolha. 
Tabelas 2B - Emigração e procura de trabalho fora de Portugal

\begin{tabular}{lcc}
\hline Especialidade & \multicolumn{2}{l}{ Simão } \\
\hline Cardiologia & $94 \%$ & $6 \%$ \\
Anatomia Patológica & $90 \%$ & $10 \%$ \\
Infecciologia & $90 \%$ & $10 \%$ \\
Oncologia Médica & $88 \%$ & $12 \%$ \\
Ortopedia & $87 \%$ & $13 \%$ \\
Neurologia & $82 \%$ & $18 \%$ \\
Cirurgia Geral & $81 \%$ & $19 \%$ \\
Oftalmologia & $80 \%$ & $20 \%$ \\
Ginecologia/Obstetrícia & $75 \%$ & $25 \%$ \\
Anestesiologia & $73 \%$ & $27 \%$ \\
Medicina Interna & $71 \%$ & $29 \%$ \\
Nefrologia & $70 \%$ & $30 \%$ \\
Otorrinolaringologia & $70 \%$ & $30 \%$ \\
Pediatria & $69 \%$ & $31 \%$ \\
Radiodiagnóstico & $67 \%$ & $33 \%$ \\
Medicina Física e Reabilitação & $64 \%$ & $36 \%$ \\
Pneumologia & $64 \%$ & $36 \%$ \\
Gastrenterologia & $61 \%$ & $39 \%$ \\
Psiquiatria & $54 \%$ & $46 \%$ \\
Medicina Geral e Familiar & $49 \%$ & $51 \%$ \\
Medicina Legal & $40 \%$ & \\
Saúde Pública & $13 \%$ & \\
Total & $65 \%$ & \\
\hline
\end{tabular}

\section{Expectativas em relação à especialidade}

A maioria dos médicos considera que as suas expectativas corresponderam muito $(48 \%)$ ou totalmente $(41 \%)$ à realidade encontrada na especialidade, $7 \%$ deram uma resposta neutra e $4 \%$ consideram que corresponderam pouco ou nada.

\section{Grau de satisfação com a especialidade}

Verifica-se que $52 \%$ dos médicos se encontram extremamente satisfeitos, $36 \%$ muito satisfeitos, $7 \%$ satisfeitos, $3 \%$ pouco satisfeitos e $2 \%$ nada satisfeitos com a sua especialidade. Quando questionados se escolheriam a mesma especialidade novamente, $91 \%$ dos Médicos ( $n=$ 550) respondeu afirmativamente.

\section{Análises por especialidade}

Da subamostra de médicos cuja especialidade foi a primeira escolha analisaram-se apenas as especialidades que contaram com dez ou mais respostas.

\section{Expectativas em relação à especialidade}

As expectativas mais elevadas encontram-se na Anestesiologia ( $M=8,97 ; D P=0,95)$, Ginecologia/Obstetrícia ( $M$ $=8,67 ; \mathrm{DP}=1,15)$ e Neurologia $(\mathrm{M}=8,3 ; \mathrm{DP}=0,67)$; seguidas de Cardiologia ( $M=8,24 ; D P=0,97)$; Radiodiagnóstico $(M=8,14 ; D P=1,29)$; Gastroenterologia $(M=8,12 ; D P=$ 1,62); Medicina Geral e Familiar ( $M=8,09$; $D P=1,63)$; Medicina Interna $(M=8,06$; $D P=1,65)$; Psiquiatria $(M=8,04$; $\mathrm{DP}=1,02)$; Ortopedia $(\mathrm{M}=7,73$; $\mathrm{DP}=2,19)$; Oncologia Médica $(M=7,71$; $D P=1,44)$ e Cirurgia Geral $(M=7,26$; $\mathrm{DP}=2,14)$.

\section{Grau de satisfação com a especialidade}

A Anestesiologia obtém novamente a média mais elevada $(M=9,03 ; D P=0,87)$, seguida pela Ginecologia/Obstetrícia $(M=8,76$; $D P=1,12)$; Neurologia $(M=8,7 ; D P=$ $0,82$ ); Psiquiatria ( $M=8,52 ; D P=0,96)$; Gastroenterologia $(\mathrm{M}=8,47 ; \mathrm{DP}=0,80)$; Radiodiagnóstico $(\mathrm{M}=8,36 ; \mathrm{DP}=$ 1,39); Cardiologia $(M=8,29$; $D P=1,10)$; Medicina Geral e Familiar ( $M=8,17$; $D P=1,73)$; Ortopedia $(M=8,14$; $D P=$ 2,32); Oncologia Médica ( $M=8$; $D P=1,47)$; Pediatria ( $M=$ 
7,97; $\mathrm{DP}=1,97)$; Medicina Interna $(\mathrm{M}=7,71$; $\mathrm{DP}=1,84) \mathrm{e}$ Cirurgia Geral $(M=6,84 ; D P=2,58)$.

\section{Perspetivas de futuro}

A maior perspetiva encontra-se na Anestesiologia ( $\mathrm{M}=$ 8,26; $D P=1,48)$, Oncologia Médica $(M=8,07 ; D P=1,27)$ e Medicina Geral e Familiar ( $M=8$; $D P=1,61$ ), Ortopedia ( $M$ = 7,73; $\mathrm{DP}=2,14)$; Radiodiagnóstico ( $\mathrm{M}=7,57 ; \mathrm{DP}=1,65)$; Medicina Interna ( $M=7,56$; $D P=1,56)$; Gastroenterologia $(M=7,41 ; D P=1,70)$; Ginecologia/Obstetrícia $(M=7,37$; $\mathrm{DP}=1,30)$; Psiquiatria $(\mathrm{M}=7,2 ; \mathrm{DP}=1,66)$; e Neurologia $(M=7,2$; $D P=1,69)$. Com menos perspetivas estão Cardiologia $(M=6,71 ; D P=1,26)$; a Cirurgia Geral $(M=5,84 ; D P$ $=1,86)$ e a Pediatria $(M=5 ; D P=2,11)$.

\section{DISCUSSÃO}

O presente artigo é o primeiro focar-se na satisfação com a especialidade entre internos da formação específica em Portugal. Os resultados sugerem que somente $10 \%$ dos internos se encontram insatisfeitos com a especialidade, enquanto que $78 \%$ referem apresentar-se muito ou mesmo extremamente satisfeitos com a sua formação. Estes resultados são comparáveis aos obtidos noutros estudos semelhantes realizados em Países Ocidentais, onde estão descritas taxas de $85 \%$ de satisfação (satisfeito ou muito satisfeito) com a sua profissão, ${ }^{7}$ sendo também concordantes com estudos efectuados entre internos de formação específica de outras nacionalidades. A literatura em Portugal é muito escassa, mas num recente estudo entre internos de Medicina Geral e Familiar da região norte de Portugal observou-se que $91 \%$ dos internos estava satis- feito ou muito satisfeito com a especialidade, corroborando os resultados observados no presente estudo. ${ }^{17}$

No entanto, embora este não seja um estudo longitudinal, observa-se uma redução da satisfação ao longo do internato, com os internos dos últimos anos a reportarem uma menor satisfação com a especialidade quando comparados com os colegas mais jovens. Esta redução pode ser devida a diversos fatores, como uma maior exigência profissional nos anos finais do internato, o desvanecer do entusiasmo inicial dos primeiros anos ou as diferenças nas condições de trabalho experienciadas pelos internos dos últimos anos, consequência da deterioração da condição económica vivida em Portugal. Será desta forma importante que se tente perceber a razão da insatisfação mas também de encontrar formas de a combater. No futuro próximo, estudos mais pormenorizados devem tentar avaliar as razões da insatisfação, enquanto que algumas acções devem ser tomadas para prevenir a insatisfação, como a realização de acções de esclarecimento, criação de comissões de internos, articulação com comissão de internato nos Hospitais, entre outros. Assim, apesar da satisfação demonstrada, é preocupante observar que $81 \%$ dos médicos considera que o panorama da prática clínica em Portugal piorou muito ou extremamente nos últimos anos, enquanto que somente $2 \%$ dos internos considera que esta atualmente melhorou. Desta forma não será de estranhar que os níveis de satisfação se reduzam num futuro próximo.

Como provável consequência desta perceção de um agravamento das condições de trabalho, $65 \%$ dos médicos internos refere que considera emigrar no final da especialidade. Estes valores elevam-se até $75 \%$ no último ano

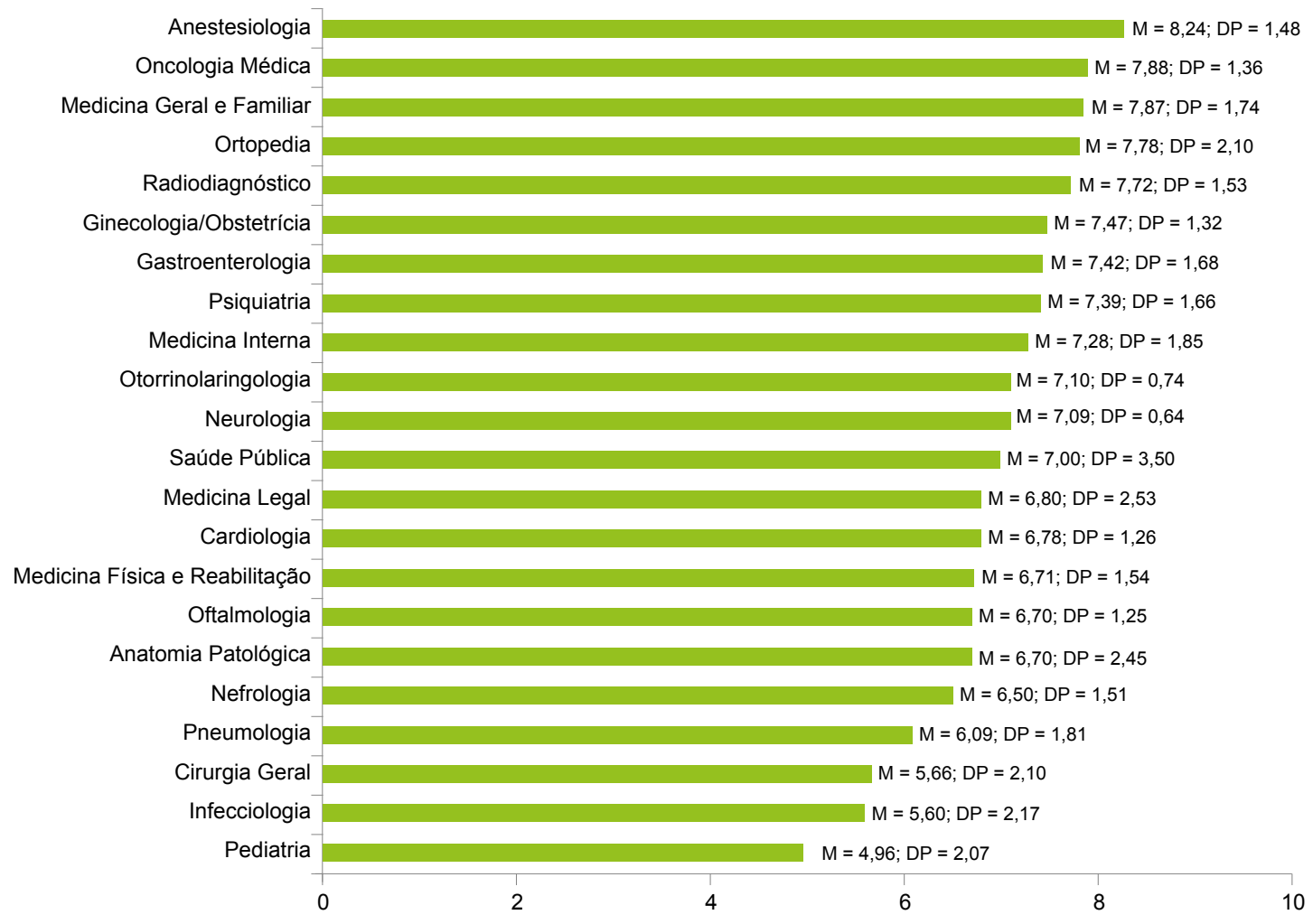

Figura 3 - Perspetivas de futuro, por especialidade 


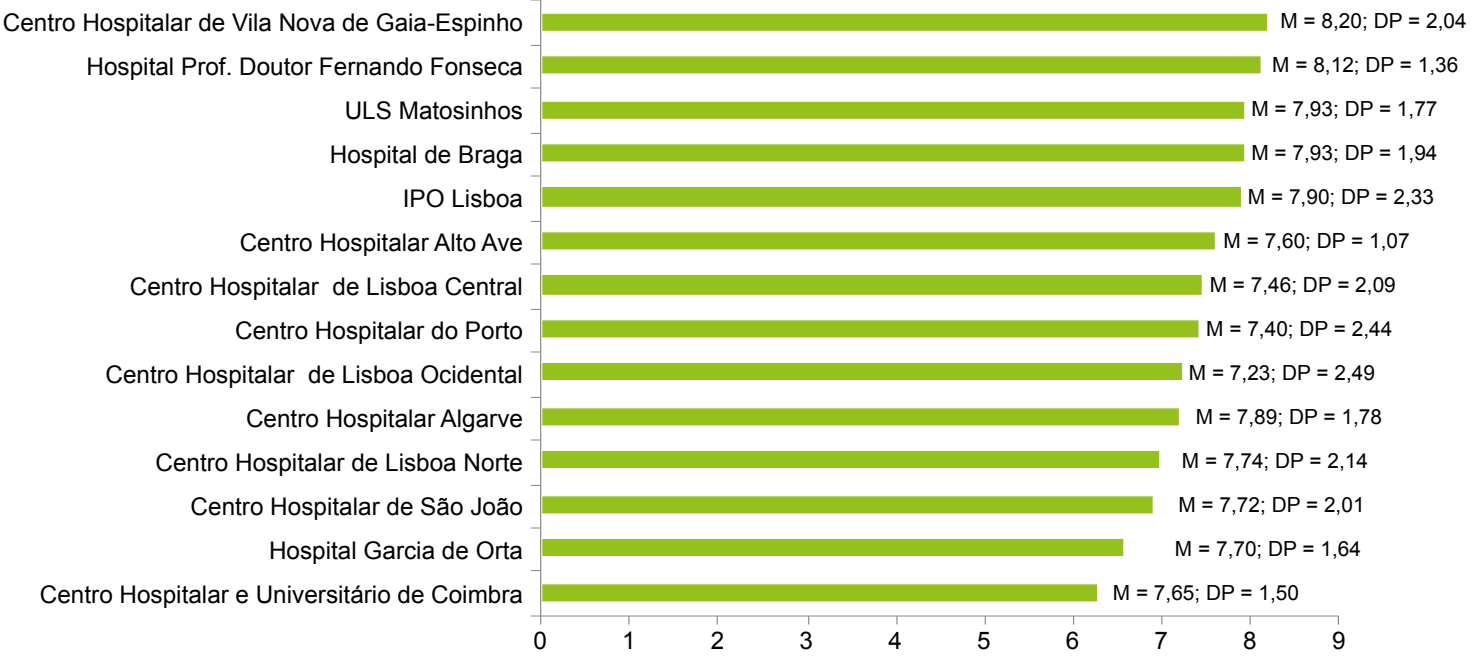

Figura 4A - Grau de satisfação com o local de formação

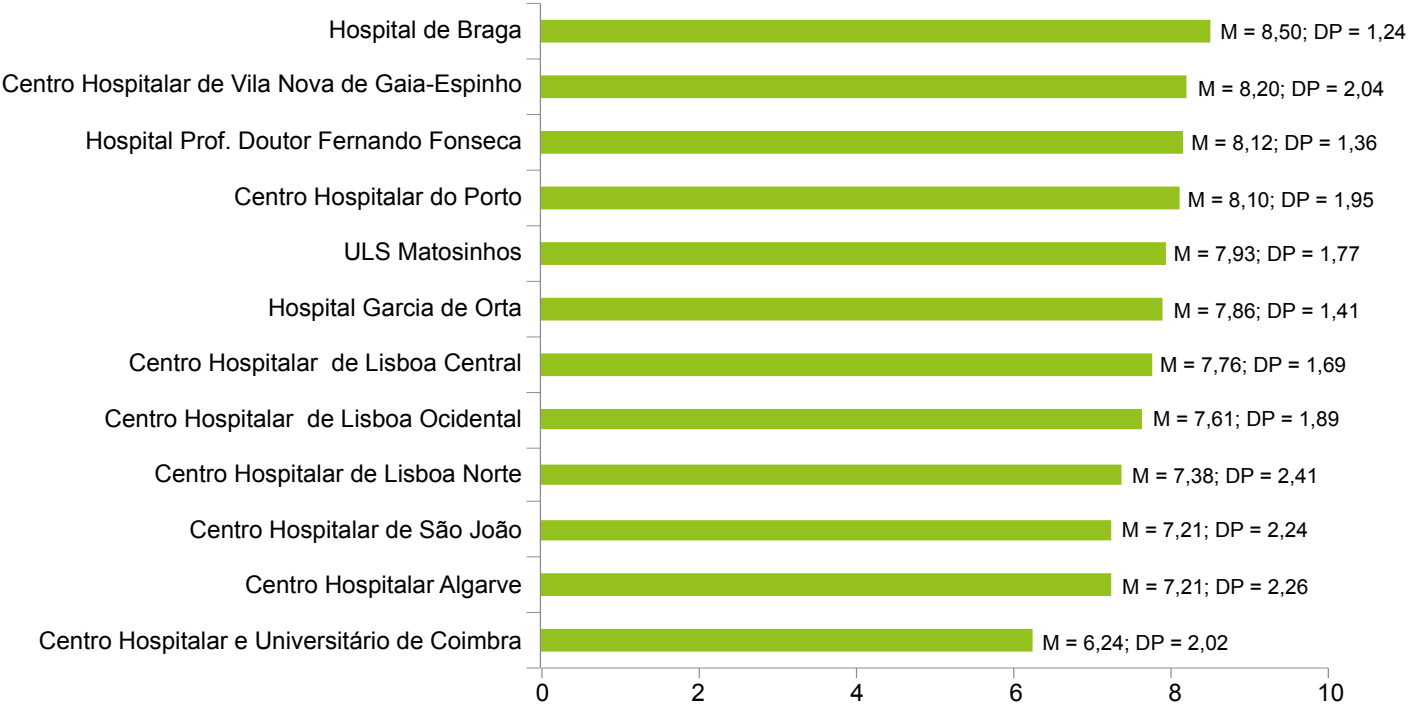

Figura 4B - Grau de satisfação com o local de formação, controlando pela satisfação

de internato, período provável da tomada dessa decisão. Estes dados são muito similares aos encontrados recentemente na Islândia, país que como Portugal foi muito afectado pela crise económica de 2008 , com $63 \%$ dos médicos islandeses ponderando emigrar num futuro próximo, tendo nesse estudo a satisfação profissional um impacto estatisticamente significativo nesta decisão. ${ }^{15}$ Tratam-se de dados com relevância significativa que, exigem reflexão na forma como a saúde deve ser gerida em Portugal. Num momento em que o País enfrenta um envelhecimento significativo do seu corpo clínico e no qual existem locais com rácios de médicos por habitante ainda bastante abaixo do necessário, a emigração de médicos poderá tornar-se não só um problema económico como também um problema de saúde pública. As implicações da emigração médica são enormes e devem gerar preocupações a nível económico e social. A formação pré e pós graduada de um interno em
Portugal pode atingir valores entre os 300 e 400000 euros, um valor demasiado elevado para ser desperdiçado com a emigração após esta formação. Socialmente, a emigração agrava ainda mais a carência de médicos em Portugal, sendo que a emigração dos jovens especialistas contribui para um acentuar dramático do envelhecimento médico já verificado neste país, ao mesmo tempo que priva a população de uma força de trabalho ambiciosa, de grande capacidade de trabalho e com contacto com as mais recentes técnicas médicas.

Por outro lado, os resultados referentes à mudança de especialidade ou mesmo de profissão médica merecem uma reflexão. Devido ao facto do curso de Medicina ser dos cursos mais desejados pelos estudantes do ensino secundário, não seria expectável que uma percentagem tão elevada de internos não considerasse escolher novamente o curso de Medicina se voltasse atrás. No entanto, 
estes dados são muito similares ao de um estudo canadiano, envolvendo 415 internos da especialidade, em que $22 \%$ considerava não escolher novamente o curso de Medicina, enquanto $15 \%$ considerava mudar de especialidade..$^{18}$ Percentagens ainda maiores foram encontradas em estudos americanos, com $30 \%$ dos médicos referindo que não escolheriam de novo a carreira médica se lhes fosse dada essa oportunidade, ${ }^{19}$ enquanto $40 \%$ não recomendariam o curso de Medicina a um jovem prestes a entrar no mundo universitário. ${ }^{20}$

Os dados que comparam as diferentes especialidades entre si são difíceis de interpretar, quer devido ao reduzido número de estudos similares quer aos resultados conflituantes. De facto, enquanto que alguns estudos mostram que são especialidades como a Radiologia que apresenta níveis de satisfação mais elevados, ${ }^{18}$ outros mostram a Dermatologia ou a Infecciologia. ${ }^{12}$ A disparidade dos nossos resultados em relação a estudos anteriores pode dever-se a vários fatores, decorrentes não só da realidade própria de Portugal como pelo facto da população estudada no nosso estudo abranger somente internos. No sentido oposto verificámos que foram as especialidades de Cirurgia Geral, Medicina Legal e Saúde Pública aquelas que apresentaram níveis de satisfação mais baixos. Dado serem especialidades cuja prática diária pouco têm em comum, não nos é possível identificar um único factor responsável pelos baixos níveis de satisfação. Podemos especular que vários fatores, como a carga horária na Cirurgia Geral ou a pouca relevância dada nos programas universitários à Saúde Pública e à Medicina Legal as razões para a este insatisfação. No entanto, até à realização de estudos mais aprofundados qualquer justificação é meramente especulativa. Da mesma forma, em agregado observa-se que são os Hospitais Centrais (como os CHUC, CH São João e Lisboa Norte) que apresentam médicos com níveis de satisfação mais baixo. Estes resultados podem ser indicativos que a formação em Hospitais de maior dimensão, em que a componente pessoal é menor e a competição é necessariamente maior, podem ser responsáveis por níveis de satisfação menores. Outro dos fatores que potencialmente pode levar a que os internos dos Hospitais Centrais sejam aqueles com níveis de satisfação mais baixo é o serviço de urgência. Os serviços de urgência dos Hospitais Centrais servem por norma uma população maior e com maior grau de complexidade, tornando o trabalho dos internos destes Hospitais mais intenso, frequente e de maior exigência, o que pode influenciar a satisfação que o interno apresenta em relação à sua formação. É importante que estes resultados tenham em conta as diferenças entre Hospitais no número de especialidades que apresentam e de que forma estes podem influenciar os resultados. Assim, enquanto que num Hospital Central o número de especialidades onde há internos é bastante significativo, num Hospital de menor dimensão os internos concentram-se num pequeno número de especialidades. Tomámos em consideração esse factor, e tentámos de alguma forma controlar pelas especialidades existentes, selecionando somente aqueles resultados acima de um determinado limiar. No entanto, esta abordagem estatística é passível de ser criticada e os níveis de satisfação entre Hospitais devem por isso ser analisados com limitações.

Apesar dos resultados obtidos, várias são as limitações deste estudo. Em primeiro lugar, a taxa de resposta, que engloba apenas $12 \%$ dos internos de Portugal. Embora reduzida é semelhante a outros estudos internacionais, sendo que o número absoluto de respostas possibilita uma análise estatística adequada. $\mathrm{O}$ facto de não ter sido contactada a totalidade dos médicos internos limitou também o número de respostas obtidas. No entanto, o facto de ter sido possível enviar o inquérito para 5788 médicos, correspondendo a $88,19 \%$ do número total de internos, torna esta amostra muito representativa. Em segundo lugar, o nosso estudo mostrou diferenças nas respostas entre os diferentes anos do internato. Devido à nossa amostra ter mais médicos dos primeiros anos de internato, e apesar de termos efectuado análises separadas por ano, os resultados globais poderiam ter sido outros caso a amostra tivesse sido mais equilibrada. Em terceiro lugar, a representatividade da amostra por especialidade apresenta algumas limitações. Apesar de terem sido analisadas apenas as especialidades que contaram com 10 ou mais respostas isto pode ainda introduzir um viés nos resultados uma vez que as especialidades variam em termos de número de colocados. Assim, por exemplo, exatamente com o mesmo número de respostas, especialidades como 'Medicina Legal' (11 respostas em 32 colocados - taxa de resposta de $34,82 \%$ ) poderão estar sobre-representadas; e especialidades como a 'Oftalmologia' (11 respostas em 117 colocados - taxa de resposta de $9,40 \%$ ) poderão estar sub-representadas. Em quarto lugar, utilizámos um questionário que não foi validado anteriormente. No entanto, não existem escalas internacionais aferidas para avaliar a satisfação com a especialidade, e a maior parte dos estudos utiliza inquéritos construídos especificamente para o efeito. Desta forma, optámos por criar e adaptar o nosso questionário a partir desses inquéritos internacionais. Por fim, o facto deste estudo se ter focado somente nos internos não permite a extrapolação para a restante população clínica.

\section{CONCLUSÕES}

A satisfação com o internato médico em Portugal é elevada, apesar das alterações na prática clínica decorrente das recentes alterações salariais e de políticas de saúde. No entanto, uma grande maioria dos internos considera que há um agravamento significativo das condições para a prática clínica em Portugal, o que pode justificar a percentagem elevada de médicos que considera emigrar no final do seu internato. 


\section{CONFLITOS DE INTERESSE}

Os autores declaram não ter qualquer conflito de interesse relativamente ao presente artigo.

\section{REFERÊNCIAS}

1. Haas JS, Cook EF, Puopolo AL, Burstin HR, Cleary PD, Brennan TA. Is the professional satisfaction of general internists associated with patient satisfaction? J Gen Intern Med. 2000;15:122-8.

2. Kravitz RL, Shapiro MF, Linn LS, Froelicher ES. Risk factors associated with participation in the Ontario, Canada doctors' strike. Am J Public Health. 1989;79:1227-33.

3. Hillhouse JJ, Adler CM, Walters DN. A simple model of stress, burnout and symptomatology in medical residents: A longitudinal study. Psychol Health Med. 2000,5:63-73.

4. Tu HT, O'Malley AS. Exodus of male physicians from primary care drives shift to specialty practice. Track Rep. 2007:1-6.

5. Cooper RA. Seeking a balanced physician workforce for the 21st century. JAMA. 1994;272:680-7.

6. Bovier PA, Perneger TV. Predictors of work satisfaction among physicians. Eur J Public Health. 2003;13:299-305.

7. Joyce CM, Schurer S, Scott A, Humphreys J, Kalb G. Australian doctors' satisfaction with their work: results from the MABEL longitudinal survey of doctors. Med J Aust. 2011;194:30-3.

8. Cohen JS, Patten S. Well-being in residency training: a survey examining resident physician satisfaction both within and outside of residency training and mental health in Alberta. BMC Med Educ. 2005;5:21.

9. Millán T, de Carvalho KM. Satisfaction with ophthalmology residency training from the perspective of recent graduates: a cross-sectional study. BMC Med Educ. 2013;13:75.

10. Rosta J, Nylenna M, Aasland OG. Job satisfaction among hospital doctors in Norway and Germany. A comparative study on national samples. Scand J Public Health. 2009;37:503-8.

11. Leigh JP, Tancredi DJ, Kravitz RL. Physician career satisfaction within specialties. BMC Health Serv Res. 2009;9:166.

\section{FONTES DE FINANCIAMENTO}

Os autores declaram não ter recebido qualquer subsídio relativo ao presente artigo.

12. Leigh JP, Kravitz RL, Schembri M, Samuels SJ, Mobley S. Physician career satisfaction across specialties. Arch Intern Med. 2002;162:157784.

13. Kaiser family foundation. National survey of physicians part III: doctor's opinion about their profession, March 2002; [consultado 2014 Out 02] Disponível em http://kff.org/health-costs/poll-finding/national-survey-ofphysicians-part-iii-doctors/.

14. Solberg IB, Tómasson K, Aasland O, Tyssen R. Cross-national comparison of job satisfaction in doctors during economic recession. Occup Med. 2014,64:595-600.

15. Solberg IB, Tómasson K, Aasland O, Tyssen R. The impact of economic factors on migration considerations among Icelandic specialist doctors: a cross-sectional study. BMC Health Serv Res. 2013;13:524.

16. IBM Corp. Released 2011. IBM SPSS Statistics for Windows, Version 20.0. New York: IBM Corp; 2011.

17. Azevedo A, Domingues B, Moura J, Santos L. Estão os internos satisfeitos com o internato de medicina geral e familiar? Rev Port Med Geral Fam. 2014;30:24-30.

18. Cohen JS, Leung Y, Fahey M, Hoyt L, Sinha R, Cailler L, et al. The happy docs study: a Canadian Association of Internes and Residents well-being survey examining resident physician health and satisfaction within and outside of residency training in Canada. BMC Res Notes. 2008;1:105.

19. Chuck JM, Nesbitt TS, Kwan J, Kam SM. Is being a doctor still fun? West J Med. 1993;159:665-9.

20. Donelan K, Blendon RJ, Lundberg GD, Calkins DR, Newhouse JP, Leape LL, et al. The new medical marketplace: physicians' views. Health Aff . 1997;16:139-48. 
Maria João MARTINS, Inês LAÍNS, Bruno BROCHADO, Manuel OLIVEIRA SANTOS, Pedro PINTO TEIXEIRA, Mariana BRANDÃO, Rui João CERQUEIRA, Ricardo CASTRO-FERREIRA, Carlos BERNARDES, Miguel NOBRE MENEZES, Bernardo SOARES BAPTISTA, Ricardo LADEIRAS-LOPES, Mariana CRUZ REI, Gilberto PIRES DA ROSA, José Luis MARTINS, Maria MENDONÇA SANCHES, Manuel J. FERREIRA-PINTO, Margarida RATO, Miguel COSTA E SILVA, Catarina POLICIANO, João BEATO, João BARBOSA-BREDA, João PIMENTEL TORRES, Inês LEAL, Sílvia AGUIAR ROSA, Bárbara CARVALHO RIBEIRO, Francisco REGO COSTA, Carolina PALMELA, Tiago CÚRDIA GONÇALVES, Luis MORAIS, Tiago REIS MARQUES

\section{Satisfação com a Especialidade entre os Internos da Formação Específica em Portugal}

Acta Med Port 2015:28:209-221

Publicado pela Acta Médica Portuguesa, a Revista Científica da Ordem dos Médicos

Av. Almirante Gago Coutinho, 151

1749-084 Lisboa, Portugal.

Tel: +351218428215

E-mail: submissao@actamedicaportuguesa.com

www.actamedicaportuguesa.com

ISSN:0870-399X | e-ISSN: 1646-0758

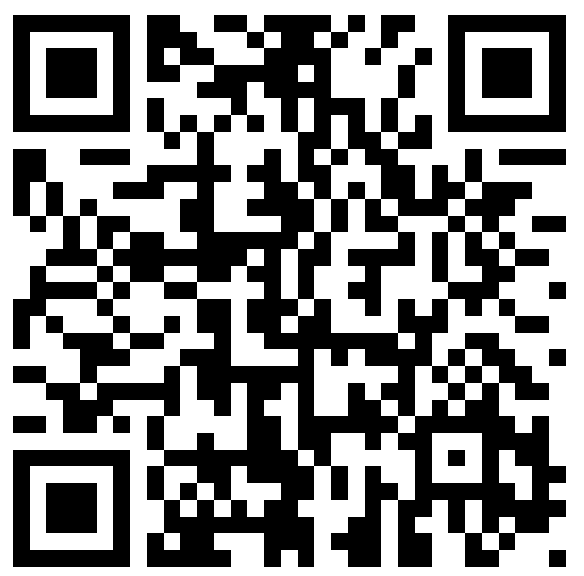

